\title{
Quantum two-state dynamics driven by stationary non-Markovian discrete noise: Exact results
}

\author{
Igor Goychuk *, Peter Hänggi \\ Universität Augsburg, Institut für Physik, Universitätsstr. 1, D-86135 Augsburg, Germany
}

Received 20 July 2005; accepted 25 November 2005

Available online 20 December 2005

\begin{abstract}
We consider the problem of stochastic averaging of a quantum two-state dynamics driven by non-Markovian, discrete noises of the continuous time random walk type (multistate renewal processes). The emphasis is put on the proper averaging over the stationary noise realizations corresponding, e.g., to a stationary environment. A two-state non-Markovian process with an arbitrary non-exponential distribution of residence times (RTDs) in its states with a finite mean residence time provides a paradigm. For the case of a two-state quantum relaxation caused by such a classical stochastic field we obtain the explicit exact, analytical expression for the averaged Laplace-transformed relaxation dynamics. In the limit of Markovian noise (implying an exponential RTD), all previously known results are recovered. We exemplify new more general results for the case of non-Markovian noise with a biexponential RTD. The averaged, real-time relaxation dynamics is obtained in this case by numerically exact solving of a resulting algebraic polynomial problem. Moreover, the case of manifest non-Markovian noise with an infinite range of temporal autocorrelation (which in principle is not accessible to any kind of perturbative treatment) is studied, both analytically (asymptotic long-time dynamics) and numerically (by a precise numerical inversion of the Laplace-transformed averaged quantum relaxation).
\end{abstract}

(C) 2005 Elsevier B.V. All rights reserved.

Keywords: Quantum dynamics; Stationary environment; Non-Markovian noise; Stochastic path averaging

\section{Introduction}

The influence of a stochastic environment on relaxation and charge transfer processes in condensed media [1-3] is a longstanding problem of prime importance in chemical and statistical physics [4-10]. In this context, exactly solvable models are rather rare. The method of a stochastic Hamiltonian by Anderson and Kubo [11-13], known also under the label of stochastic Liouville equation (SLE) approach [14-22] presents a common methodology that has been employed over decades [10,22,23]: It is based on the simplifying representation of a stochastic environment by a classical stochastic field that acts on the quantum system of interest without a feedback mechanism. This set up thus necessarily inherits some shortcomings. In particular,

\footnotetext{
${ }^{*}$ Corresponding author.

E-mail address: goychuk@physik.uni-augsburg.de (I. Goychuk).
}

quantum states become asymptotically populated with equal weight as if the environment temperature were infinite. This shortcoming can be cured in an ad hoc manner by adding an extra term to the stochastically averaged Liouville equation to ensure a proper thermal equilibrium. What "proper" means, however, is specific to the problem under consideration. Therefore, the mentioned shortcoming can be overcome rigorously only within a full quantum-mechanical treatment of the total, coupled system-environment dynamics $[24,25]$. Notwithstanding this principal difficulty, the SLE approach remains useful and popular over the years. The method is particularly appealing because it allows to extract exact results for a number of interesting physical models [17,20,22,23,26-29].

In view of the central limit theorem, the use of classical random forces with a Gaussian statistics is most frequently employed for this sort of semiclassical modeling. The case of Gaussian white noise serves frequently as a simple model 
for the corresponding classical stochastic bath; it formally corresponds to a heat bath with an infinite spectrum of excitations. For this noise model there exists a number of exact results $[17,20,22,23,26,29]$. Realistic thermal baths possess, however, a spectrum with cut-off at finite energies. This circumstance then renders temporal autocorrelations that decay on a finite time scale. The use of stationary Gaussian-Markov noise with an exponentially decaying temporal autocorrelations (Ornstein-Uhlenbeck process) presents one of the simplest models for the corresponding colored noise $[20,30]$. We remark, however, that even for the archetype case of a two-state tunneling system this colored noise model cannot be solved exactly, except for some special situations, see, e.g., in Ref. [27] for a stochastic Landau-Zener model. In practice, one is forced to invoke additional approximations; such as in the case of a weakly colored noise the method of cumulant expansions $[20,21,31]$. It is equivalent to an expansion in a small parameter (the Kubo number) which is the root mean square (rms) of the fluctuations of the characteristic coupling energy (in frequency units) times the autocorrelation time of corresponding bath fluctuations [31]. Other perturbation schemes can also be used [30]. In particular, the opposite limit of large Kubo numbers (quasi-static fluctuations) also allows for a consistent perturbative treatment [30-32].

Non-perturbative approaches are, however, much more appealing. Continuous state fluctuations can be approximated with jump-like stochastic processes possessing a large number of discrete states. An example presents a discretization of the spatial degree of freedom of a continuous diffusion process. The class of Markovian discrete state noises provide a rather general framework for a formally exact averaging without using any kind of perturbation expansion [13,16,33,36-39]. The stochastic path integral approach $[34,35]$, adapted for such jump processes $[16,33]$ is particularly convenient. There exist yet other powerful approaches, cf. in Refs. [36,40-43]. The two-state Markovian noise (or dichotomous noise) presents the archetype discrete state process which allows for an exact study of stochastically driven two-level quantum systems [40,4450]. Furthermore, the multistate case of an exciton transfer in molecular aggregates with many independent noise sources modeled by independent two-state Markovian noises is also solvable, in the sense that it can be reduced to solving numerically an algebraic relation [51]. This dichotomous noise can serve to model a pseudo-spin $1 / 2$ stochastic bath degree of freedom. In the case of electron transfer in molecular systems such pseudo-spin stochastic variables can simulate, for example, the bistable fluctuations of a charged molecular group nearby the donor, or the acceptor site, or also the conformational fluctuations of a bistable molecular bridge [52].

The method of a stochastic path averaging can readily be generalized onto non-Markovian jump processes of the renewal (or continuous time random walk) type [5355]. Such processes are then characterized by the set of res- idence time distributions (RTDs) $\psi_{j}(\tau)$ in the noise states $j$ and the probabilities $p_{i j}$ for undergoing a transition from state $j \rightarrow i$ at the end of each residence time interval (RTI) into another noise state $i$. The lengths of subsequent RTIs are mutually independent. ${ }^{1}$ This constitutes the crucial ingredient that allows for an exact averaging for such non-Markovian processes [53]. Attention must be paid, however, to the stationarity of the process, i.e., to the proper averaging over the stationary noise realizations in a stationary environment. This issue is not trivial and requires special consideration: Such a stationary averaging is possible only if the average residence times are finite for all discrete noise states. The stationary noise averaging can be performed exactly in the Laplace-domain for any nonMarkovian process of the considered type and arbitrary quantum dynamics [54,57]. In particular, the Laplacetransform of the corresponding quantum propagator can be written in a general analytical form [57]. It yields a complex expression which practically can be elaborated on explicitly in some special cases only. In this work, we present analytical expressions for averaged quantum two-state dynamics driven by a symmetric two-state non-Markovian noise with arbitrary RTD possessing a finite mean time. Our results generalize the prior results of Refs. [40,44,50] and reduce to the latter ones in the particular case of Markovian noise.

The paper is structured as follows. We first review the general approach and present the main result for the Laplace-transformed stationary-averaged quantum propagator in Section 2. A specific application of this general result to the Kubo oscillator is given in Section 3. Explicit results for a quantum two-state dynamics driven by a twostate non-Markovian noise are provided in Section 4. The limit of Markovian noise and a special non-Markovian case are studied for the two-state quantum dynamics in Section 5. The case of manifest non-Markovian noise with infinite mean autocorrelation time is considered in Section 6. In Section 7 we provide a resume of our results.

\section{Stochastic Liouville-von-Neumann equation and corresponding averaged quantum propagator}

To start out, let us consider an arbitrary quantum system with a Hamilton operator $\hat{H}[\xi(t)]$ which depends on a classical, noisy parameter $\xi(t)$. This stochastic process $\xi(t)$ can take on either continuous or discrete values. Accordingly, the Hamiltonian $\hat{H}$ randomly in time acquires

\footnotetext{
${ }^{1}$ This does not necessarily imply that the process is Markovian: If the RTD of some state is non-exponential the process is clearly nonMarkovian and exhibits memory effects. This can be understood due to the following reasoning which can be traced back to Ref. [56]. For a memoryless, Markovian process the survival probability $\Phi_{j}(\tau):=$ $\int_{\tau}^{\infty} \psi_{j}\left(\tau^{\prime}\right) \mathrm{d} \tau^{\prime}$ in any state $j$ must obviously assume the following property: $\Phi_{j}\left(\tau_{1}+\tau_{2}\right)=\Phi_{j}\left(\tau_{1}\right) \Phi_{j}\left(\tau_{2}\right)$. The only non-trivial solution of this latter functional equation reads $\Phi_{j}(\tau)=\exp \left(-\gamma_{j} \tau\right)$, where $\gamma_{j}>0$ is the rate to leave the state $j$
} 
different operator values $\hat{H}[\xi(t)]$ which generally do not commute, i.e., $\left[\hat{H}[\xi(t)], \hat{H}\left[\xi\left(t^{\prime}\right)\right]\right] \neq 0$.

The prime objective is then to noise-average the corresponding quantum dynamics which is characterized by the Liouville-von-Neumann equation

$\frac{\mathrm{d}}{\mathrm{d} t} \rho(t)=-\mathrm{i} \mathscr{L}[\xi(t)] \rho(t)$

for the density operator $\rho(t)$ over the realizations of the noise $\xi(t) . \mathscr{L}[\xi(t)]$ in Eq. (1) denotes the quantum Liouville superoperator, $\mathscr{L}[\xi(t)](\cdot)=\frac{1}{\hbar}[\hat{H}[\xi(t)],(\cdot)]$. In other words, the posed challenge consists in evaluating the noise-averaged propagator

$\left\langle S\left(t_{0}+t, t_{0}\right)\right\rangle=\left\langle\mathscr{T} \exp \left[-\mathrm{i} \int_{t_{0}}^{t_{0}+t} \mathscr{L}[\xi(\tau)] \mathrm{d} \tau\right]\right\rangle$,

where $\mathscr{T}$ denotes the time-ordering operator.

\subsection{Non-Markovian vs. Markovian discrete state fluctuations}

We next specify this task for noise assuming $N$ discrete states $\left\{\xi_{i}\right\}$. The noise is generally assumed to present a non-Markovian renewal process which is fully characterized by the set of transition probability densities $\psi_{i j}(\tau)$ for making random transitions within the time interval $[\tau, \tau+\mathrm{d} \tau]$ from the state $j$ to the state $i$. These probability densities are necessarily positive and obey the normalization conditions

$\sum_{i=1}^{N} \int_{0}^{\infty} \psi_{i j}(\tau) \mathrm{d} \tau=1$

for all $j=1,2, \ldots, N$.

The subsequent residence time-intervals between jumps are assumed to be mutually uncorrelated. The residence time distribution (RTD) $\psi_{j}(\tau)$ in the state $j$ reads

$\psi_{j}(\tau)=\sum_{i} \psi_{i j}(\tau)=-\frac{\mathrm{d} \Phi_{j}(\tau)}{\mathrm{d} t}$.

The survival probability $\Phi_{j}(\tau)$ of the state $j$ follows then as

$\Phi_{j}(\tau)=\int_{\tau}^{\infty} \psi_{j}(\tau) \mathrm{d} \tau$.

This constitutes the general scheme for continuous time random walk (CTRW) theory [58-61].

Several particular descriptions used for such non-Markovian processes of the renewal type are worth mentioning. The approach in Ref. [53] in terms of time-dependent aging rates $k_{i j}(t)$ for the transitions from state $j$ to state $i$ corresponds to a particular choice, reading

$\psi_{i j}(\tau):=k_{i j}(\tau) \exp \left[-\sum_{i} \int_{0}^{\tau} k_{i j}(t) \mathrm{d} t\right]$.

The Markovian case corresponds to time-independent transition rates $k_{i j}(\tau)=$ constant. Any deviation of $\psi_{i j}(\tau)$ from this Markovian case then in turn yields a time-dependence of the transition rates $k_{i j}(\tau)$ which amounts to a non-Markovian behavior. Furthermore, the survival probability $\Phi_{j}(\tau)$ in the state $j$ is determined by

$\Phi_{j}(\tau)=\exp \left[-\sum_{i=1}^{N} \int_{0}^{\tau} k_{i j}(t) \mathrm{d} t\right]$

and Eq. (6) can be recast as

$\psi_{i j}(\tau):=k_{i j}(\tau) \Phi_{j}(\tau)$.

The introduction of such time-dependent "aging" rates presents one possibility to describe non-Markovian memory effects; it is not unique though. A different scheme follows by defining [55]:

$\psi_{i j}(\tau):=p_{i j}(\tau) \psi_{j}(\tau)$

with $\sum_{i} p_{i j}(\tau)=1$. The physical interpretation is as follows: The process remains in a state $j$ for a random time interval characterized by the probability density $\psi_{j}(\tau)$. At the end of this time interval, it jumps into another state $i$ with a generally time-dependent conditional probability $p_{i j}(\tau)$. Such an interpretation is frequently used in the continuous time random walk theory. Evidently, any process of the considered type can be interpreted in this way. By equating Eqs. (8) and (9) and taking into account that $\psi_{j}(\tau):=-\mathrm{d} \Phi_{j}(\tau) / \mathrm{d} \tau$ one deduces that the approach in Ref. [53] can be related to that in Ref. [55] with the time-dependent transition probabilities

$p_{i j}(\tau)=\frac{k_{i j}(\tau)}{\sum_{i} k_{i j}(\tau)}$

and with the non-exponential probability densities $\psi_{j}(\tau)$, i.e., $\psi_{j}(\tau)=\gamma_{j}(\tau) \exp \left[-\int_{0}^{\tau} \gamma_{j}(t) \mathrm{d} t\right]$ with $\gamma_{j}(\tau):=\sum_{i} k_{i j}(\tau)$.

The description of non-Markovian effects with the timedependent transition probabilities $p_{i j}(\tau)$ is rather difficult to derive from the sample trajectories of an experimentally observed random process $\xi(t)$. The same holds true for the concept of time-dependent rates. These rates cannot be measured directly from the set of stochastic sample trajectories. On the contrary, the RTD $\psi_{j}(\tau)$ and the time-independent $p_{i j}$ (with $p_{i i}:=0$ ) can routinely be deduced from measured sample trajectories, say, in a single-molecular experiment. The study of the statistics of the residence time-intervals allows one to obtain the corresponding probability densities $\psi_{j}(\tau)$ and, hence, the survival probabilities $\Phi_{j}(\tau)$. Furthermore, the statistics of the transitions from one state into all other states allows one to derive the corresponding conditional probabilities $p_{i j}$. From this primary information, a complementary interpretation of experimental data in terms of time-dependent rates $k_{i j}(\tau)$ can readily be given as

$k_{i j}(\tau)=-p_{i j} \frac{\mathrm{d} \ln \left[\Phi_{j}(\tau)\right]}{\mathrm{d} \tau}$

if one prefers this particular "language" to describe the non-Markovian effects. Moreover, the description with a constant set $p_{i j}$ provides a consistent approach to describe 
stationary realizations of $\xi(t)$, and consequently to find the corresponding averaged quantum evolution [57]. It is this reasoning that we shall follow in the following.

\subsection{Averaging the quantum propagator}

The task of performing the noise-averaging of the quantum dynamics in Eq. (2) can be solved exactly because we can make use of the piecewise constant character of the noise realizations $\xi(t)[16,33,53]$. Indeed, let us consider the time-interval $\left[t_{0}, t\right]$ and let us take a frozen realization of $\xi(t)$ assuming $k$ switching events within this time-interval at the time-instants $t_{i}$,

$t_{0}<t_{1}<t_{2}<\cdots<t_{k}<t$.

Correspondingly, the noise takes on the values $\xi_{j_{0}}, \xi_{j_{1}}, \ldots, \xi_{j_{k}}$ in the time sequel. Then, the propagator $S\left(t, t_{0}\right)$ reads

$S\left(t, t_{0}\right)=\mathrm{e}^{-\mathrm{i} \mathscr{L}\left[\xi_{j_{k}}\right]\left(t-t_{k}\right)} \mathrm{e}^{-\mathrm{i} \mathscr{L}\left[\xi_{j_{k-1}}\right]\left(t_{k}-t_{k-1}\right)} \cdots \mathrm{e}^{-\mathrm{i} \mathscr{L}\left[\xi_{j}\right]\left(t_{1}-t_{0}\right)}$.

Let us further assume that the process $\xi(t)$ has been prepared in the state $j_{0}$ at $t_{0}$. Then, the corresponding $k$-times probability density for this noise realization reads

$$
\begin{aligned}
& P_{k}\left(\xi_{j_{k}}, t_{k} ; \xi_{j_{k-1}}, t_{k-1} ; \ldots ; \xi_{j_{1}}, t_{1} \mid \xi_{j_{0}}, t_{0}\right) \\
& \quad=\Phi_{j_{k}}\left(t-t_{k}\right) \psi_{j_{k} j_{k-1}}\left(t_{k}-t_{k-1}\right) \cdots \psi_{j_{1} j_{0}}\left(t_{1}-t_{0}\right)
\end{aligned}
$$

for $k \neq 0$ and $P_{0}\left(\xi_{j_{0}}, t_{0}\right)=\Phi_{j_{0}}\left(t-t_{0}\right)$ for $k=0$. In order to obtain the noise-averaged propagator $\left\langle S\left(t \mid t_{0}, j_{0}\right)\right\rangle$ conditioned on such a non-stationary initial noise preparation in the state $j_{0}$ one has to average (13) with the probability measure in (14) (for $k=\overline{0, \infty}$ ). This task can be readily be performed by use of the Laplace-transform method [it will be denoted in the following as $\tilde{A}(s):=\int_{0}^{\infty} \exp (-s \tau) A(\tau) \mathrm{d} \tau$ for any time-dependent quantity $A(\tau)]$. The result for $\left\langle\tilde{S}\left(s \mid t_{0}, j_{0}\right)\right\rangle=\int_{0}^{\infty} \exp (-s \tau)\left\langle S\left(t_{0}+\tau \mid t_{0}, j_{0}\right)\right\rangle \mathrm{d} \tau$ thus reads $[53,57]$

$\left\langle\tilde{S}\left(s \mid t_{0}, j_{0}\right)\right\rangle=\sum_{i}\left(\tilde{A}(s)[I-\tilde{B}(s)]^{-1}\right)_{i j_{0}}$,

where the matrix-superoperators $\tilde{A}(s)$ and $\tilde{B}(s)$ read in components:

$$
\begin{aligned}
& \tilde{A}_{k l}(s):=\delta_{k l} \int_{0}^{\infty} \Phi_{l}(\tau) \mathrm{e}^{-\left(s+\mathrm{i} \mathscr{L}\left[\xi_{l}\right]\right) \tau} \mathrm{d} \tau, \\
& \tilde{B}_{k l}(s):=\int_{0}^{\infty} \psi_{k l}(\tau) \mathrm{e}^{-\left(s+\mathrm{i} \mathscr{L}\left[\xi_{l}\right]\right) \tau} \mathrm{d} \tau,
\end{aligned}
$$

correspondingly, and $I$ denotes the unit matrix.

To obtain the stationary noise average of the propagator it necessary to average (15) over the stationary initial probabilities $p_{j_{0}}^{\text {st }}$. The averaging over the initial distribution alone is, however, not sufficient to arrive at the stationary noise-averaging in the case of non-Markovian processes since the noise realizations constructed in the way still are generally not stationary. This principal problem is rooted in the following observation: By preparing the quantum system at $t_{0}=0$ in a non-equilibrium state $\rho(0)$, the noise is taken at random in some initial state $\xi_{j_{0}}$ with the probability $p_{j_{0}}^{\text {st }}$ (stationary noise). However, every time when we repeat the preparation of the quantum system in its initial state, the noise will already occupy a (random) state $\xi_{j_{0}}$ for some unknown, random time interval $\tau_{j_{0}}^{*}$ (setting a clock at $t_{0}=0$ sets the initial time for the quantum system, but not for the noise, which is assumed to start in the infinite past). Therefore, in a stationary setting a proper averaging over this unknown time $\tau_{j_{0}}^{*}$ is necessary. The corresponding procedure implies that the mean residence time $\left\langle\tau_{j}\right\rangle$ is finite, $\left\langle\tau_{j}\right\rangle \neq \infty$, and yields a different residence time distribution for the initial noise state, $\psi_{j}^{(0)}(\tau)$; namely, it is evaluated to read $\psi_{j}^{(0)}(\tau)=\Phi_{j}(\tau) /\left\langle\tau_{j}\right\rangle$ [62]. Only for Markovian processes where $\Phi_{j}(\tau)$ is strictly exponential, does $\psi_{j}^{(0)}(\tau)$ coincide with $\psi_{j}(\tau)$. Using this $\psi_{j}^{(0)}(\tau)$ instead of $\psi_{j}(\tau)$ for the first sojourn in the corresponding state and for the time-independent set $p_{i j}$, the noise realizations become stationary [54,57,62]. The corresponding expression for the quantum propagator averaged over such stationary noise realizations has been derived in Ref. [57], cf. Eqs. (25), (29) therein. In a slightly more general form it reads

$$
\begin{aligned}
\langle\tilde{S}(s)\rangle= & \langle\tilde{S}(s)\rangle_{\text {static }}-\sum_{i j}\left(\tilde{C}(s)-\tilde{A}(s)[I-P \tilde{D}(s)]^{-1}\right. \\
& \times P \tilde{A}(s))_{i j} \frac{p_{j}^{\text {st }}}{\left\langle\tau_{j}\right\rangle},
\end{aligned}
$$

where $\langle\tilde{S}(s)\rangle_{\text {static }}$ is the Laplace-transform of the statically averaged Liouville propagator

$\langle S(\tau)\rangle_{\text {static }}:=\sum_{k} \mathrm{e}^{-\mathrm{i} \mathscr{L}\left[\xi_{k}\right] \tau} p_{k}^{\text {st }}$,

$p_{j}^{\text {st }}=\lim _{t \rightarrow \infty} p_{j}(t)$ are the stationary probabilities of noise states which are determined by a system of linear algebraic equations [54,57],

$\frac{p_{j}^{\mathrm{st}}}{\left\langle\tau_{j}\right\rangle}=\sum_{n} p_{j n} \frac{p_{n}^{\mathrm{st}}}{\left\langle\tau_{n}\right\rangle}$,

and $P$ is the matrix of transition probabilities $p_{i j}$, i.e., the "scattering matrix" of the random process $\xi(t)$. Furthermore, the auxiliary matrix operators $\tilde{C}(s)$ and $\tilde{D}(s)$ in (18) read in components:

$\tilde{C}_{k l}(s):=\delta_{k l} \int_{0}^{\infty} \mathrm{e}^{-\left(s+\mathrm{i} \mathscr{L}\left[\xi_{\xi}\right]\right) \tau} \int_{0}^{\tau} \Phi_{l}\left(\tau^{\prime}\right) \mathrm{d} \tau^{\prime} \mathrm{d} \tau$,

$\tilde{D}_{k l}(s):=\delta_{k l} \int_{0}^{\infty} \psi_{l}(\tau) \mathrm{e}^{-\left(s+\mathrm{i} \mathscr{L}\left[\xi_{l}\right]\right) \tau} \mathrm{d} \tau$.

We remark here that this very same averaging procedure can be applied to any system of linear stochastic differential equations.

\section{An archetype case: the Kubo oscillator}

A simple but very instructive application of this general procedure is the noise-averaging of the Kubo oscillator $[13,60]$; reading 
$\dot{x}(t)=\mathrm{i} \epsilon[\xi(t)] x(t)$.

This particular equation emerges in various situations such as in the theory of optical line shapes, nuclear magnetic resonance $[11,13]$, and also for single molecule spectroscopy [63]. In the context of the stochastic theory of spectral line shapes $[11,13,63], \epsilon[\xi(t)]$ in Eq. (23) corresponds to a stochastically modulated frequency of quantum transitions between the levels of a "two-state atom", or transitions between the eigenstates of a spin $1 / 2$ system.

The spectral line shape is determined via the corresponding stochastically averaged propagator of the Kubo oscillator as [13]

$I(\omega)=\frac{1}{\pi} \lim _{\eta \rightarrow+0} \operatorname{Re}[\tilde{S}(-\mathrm{i} \omega+\eta)]$.

Note that the limit $\eta \rightarrow+0$ in Eq. (24) is necessary for the regularization of the corresponding integral in the quasistatic limit $\left\langle\tau_{j}\right\rangle \rightarrow \infty$. Upon identifying $\mathscr{L}\left[\xi_{k}\right]$ with $-\epsilon_{k}$ in Eq. (18) we end up with

$$
\begin{aligned}
\langle\tilde{S}(s)\rangle= & \sum_{k} \frac{p_{k}^{\mathrm{st}}}{s-\mathrm{i} \epsilon_{k}}-\sum_{k} \frac{1-\tilde{\psi}_{k}\left(s-\mathrm{i} \epsilon_{k}\right)}{\left(s-\mathrm{i} \epsilon_{k}\right)^{2}} \frac{p_{k}^{\mathrm{st}}}{\left\langle\tau_{k}\right\rangle} \\
& +\sum_{n, l, m} \frac{1-\tilde{\psi}_{l}\left(s-\mathrm{i} \epsilon_{l}\right)}{s-\mathrm{i} \epsilon_{l}}\left(\frac{1}{I-P \tilde{D}(s)}\right)_{l m} \\
& \times p_{m n} \frac{1-\tilde{\psi}_{n}\left(s-\mathrm{i} \epsilon_{n}\right)}{s-\mathrm{i} \epsilon_{n}} \frac{p_{n}^{\mathrm{st}}}{\left\langle\tau_{n}\right\rangle}
\end{aligned}
$$

with $\tilde{D}_{n m}(s)=\delta_{n m} \tilde{\psi}_{m}\left(s-\mathrm{i} \epsilon_{m}\right){ }^{2}$ The corresponding line shape follows from Eq. (25) by virtue of Eq. (24). This result presents a non-Markovian generalization of the pioneering result by Kubo [13] for arbitrary $N$-state discrete Markovian processes. This generalization applies to arbitrary non-exponential RTDs $\psi_{k}(\tau)$, or, equivalently, in accordance with Eq. (11) also for time-dependent transition rates $k_{i j}(\tau)$. This generalization was put forward originally in Ref. [57] for a particular case, $p_{j}^{\text {st }}=\left\langle\tau_{j}\right\rangle / \sum_{k}\left\langle\tau_{k}\right\rangle$, which corresponds to an ergodic process with uniform mixing (meaning that in a long-time limit each state $j$ is visited equally often).

Next we apply this result to the case of two-state nonMarkovian noise with $p_{12}=p_{21}=1$ and $p_{1,2}^{\text {st }}=\left\langle\tau_{1,2}\right\rangle /$ $\left[\left\langle\tau_{1}\right\rangle+\left\langle\tau_{2}\right\rangle\right]$. Eq. (25) then yields

$$
\begin{aligned}
\langle\tilde{S}(s)\rangle= & \sum_{k=1,2} \frac{1}{s-\mathrm{i} \epsilon_{k}} \frac{\left\langle\tau_{k}\right\rangle}{\left\langle\tau_{1}\right\rangle+\left\langle\tau_{2}\right\rangle} \\
& +\frac{\left(\epsilon_{1}-\epsilon_{2}\right)^{2}}{\left(\left\langle\tau_{1}\right\rangle+\left\langle\tau_{2}\right\rangle\right)\left(s-\mathrm{i} \epsilon_{1}\right)^{2}\left(s-\mathrm{i} \epsilon_{2}\right)^{2}} \\
& \times \frac{\left[1-\tilde{\psi}_{1}\left(s-\mathrm{i} \epsilon_{1}\right)\right]\left[1-\tilde{\psi}_{2}\left(s-\mathrm{i} \epsilon_{2}\right)\right]}{1-\tilde{\psi}_{1}\left(s-\mathrm{i} \epsilon_{1}\right) \tilde{\psi}_{2}\left(s-\mathrm{i} \epsilon_{2}\right)} .
\end{aligned}
$$

\footnotetext{
${ }^{2}$ Note that the formal solution of another important problem, namely the (first order) relaxation kinetics with a fluctuating rate, $\dot{p}(t)=-\Gamma[\xi(t)] p(t)$ follows immediately from (25) upon substitution $\epsilon_{j} \rightarrow \mathrm{i} \Gamma_{j}$, see in Ref. [64] containing some non-trivial non-Markovian examples.
}

With (26) substituted into (24) one finds the result for the corresponding spectral line shape which matches that presented recently in Ref. [65] derived therein by use of a different method. Moreover, in the simplest case of Markovian two-state fluctuations with $\psi_{1,2}(s)=1 /(1+$ $\left.\left\langle\tau_{1,2}\right\rangle s\right)$ and with zero mean, $\langle\xi(t)\rangle=\left\langle\tau_{1}\right\rangle \epsilon_{1}+\left\langle\tau_{2}\right\rangle \epsilon_{2}=0$, this result simplifies further to read

$\langle\tilde{S}(s)\rangle=\frac{s+2 \chi}{s^{2}+2 \chi s+\sigma^{2}}$.

In $\quad(27), \quad \sigma=\sqrt{\left\langle\xi^{2}(t)\right\rangle}=\left|\epsilon_{2}-\epsilon_{1}\right| \sqrt{\left\langle\tau_{1}\right\rangle\left\langle\tau_{2}\right\rangle} /\left(\left\langle\tau_{1}\right\rangle+\left\langle\tau_{2}\right\rangle\right)$ denotes the root mean squared (rms) amplitude of fluctuations. Moreover, $\chi=v / 2+\mathrm{i} \sigma \sinh (b / 2)$ denotes a complex frequency parameter, where $v=1 /\left\langle\tau_{1}\right\rangle+1 /\left\langle\tau_{2}\right\rangle$ is the inverse of the autocorrelation time of the considered process. Its autocorrelation function reads $\left\langle\xi(t) \xi\left(t^{\prime}\right)\right\rangle=\sigma^{2} \exp$ $\left(-v\left|t-t^{\prime}\right|\right)$. Furthermore, $b=\ln \left(\left\langle\tau_{1}\right\rangle /\left\langle\tau_{2}\right\rangle\right)=\ln \left|\epsilon_{2} / \epsilon_{1}\right|$ is an asymmetry parameter. The spectral line shape corresponding to (27) has been obtained by Kubo, reading $[13,66]$,

$I(\omega)=\frac{1}{\pi} \frac{\sigma^{2} v}{\left(\omega+\epsilon_{1}\right)^{2}\left(\omega+\epsilon_{2}\right)^{2}+\omega^{2} v^{2}}$.

Moreover, the expression (27) can readily be inverted into its time domain. Note that the corresponding averaged propagator $\langle S(t)\rangle$ of Kubo oscillator [67], i.e.,

$\langle S(t)\rangle=\mathrm{e}^{-\chi t}\left[\cos \left(\sqrt{\sigma^{2}-\chi^{2}} t\right)+\frac{\chi}{\sqrt{\sigma^{2}-\chi^{2}}} \sin \left(\sqrt{\sigma^{2}-\chi^{2}} t\right)\right]$

is complex-valued when the process $\xi(t)$ is asymmetric, i.e., $b \neq 0$. This is in accordance with the asymmetry of the corresponding spectral line shape, $I(-\omega) \neq I(\omega)$. Derived in a different context [68] for the case of a two-state Markovian process with a non-vanishing mean and in quite different notation an expression equivalent to (29) is known in the theory of single-molecule spectroscopy [68-70]. For a symmetric dichotomous process (with $b=0$ ) Eq. (29) reduces to the expression (6.10) (with $\left.\omega_{0}=0\right)$ in Ref. [31].

\section{Averaged dynamics of a two-level quantum systems driven by two-state noise}

Our non-Markovian stochastic theory of quantum relaxation can be further exemplified for the relevant case of a two-state quantum system, reading

$H(t)=E_{1}|1\rangle\left\langle 1\left|+E_{2}\right| 2\right\rangle\langle 2|+\frac{1}{2} \hbar \xi(t)(|1\rangle\langle 2|+| 2\rangle\langle 1|)$,

which is driven by a two-state non-Markovian stochastic noise $\xi(t)=\left\{\Delta_{+}, \Delta_{-}\right\}$with corresponding RTDs $\psi_{+}(\tau)$, $\psi_{-}(\tau)$ and the stationary state probabilities $p_{ \pm}^{\text {st }}=\left\langle\tau_{ \pm}\right\rangle /$ $\left[\left\langle\tau_{+}\right\rangle+\left\langle\tau_{-}\right\rangle\right]$. This noise assumes the normalized stationary autocorrelation function $k(t):=\langle\delta \xi(t) \delta \xi(0)\rangle_{\mathrm{st}} /\left\langle[\delta \xi]^{2}\right\rangle_{\mathrm{st}}$ where $\delta \xi(t):=\xi(t)-\langle\xi\rangle_{\mathrm{st}}$ is temporal fluctuation. Its Laplace-transform reads [71-74] 
$\tilde{k}(s)=\frac{1}{s}-\left(\frac{1}{\left\langle\tau_{+}\right\rangle}+\frac{1}{\left\langle\tau_{-}\right\rangle}\right) \frac{1}{s^{2}} \frac{\left(1-\tilde{\psi}_{+}(s)\right)\left(1-\tilde{\psi}_{-}(s)\right)}{1-\tilde{\psi}_{+}(s) \tilde{\psi}_{-}(s)}$.

This dichotomic noise possesses the power spectrum $S_{N}(\omega)$

$S_{N}(\omega)=\frac{2\left(\Delta_{+}-\Delta_{-}\right)^{2}}{\left\langle\tau_{+}\right\rangle+\left\langle\tau_{-}\right\rangle} \frac{1}{\omega^{2}} \operatorname{Re}\left[\frac{\left(1-\tilde{\psi}_{+}(\mathrm{i} \omega)\right)\left(1-\tilde{\psi}_{-}(\mathrm{i} \omega)\right)}{1-\tilde{\psi}_{+}(\mathrm{i} \omega) \tilde{\psi}_{-}(\mathrm{i} \omega)}\right]$.

It causes (dipole) transitions between two states, $|1\rangle$ and $|2\rangle$, and is zero on average (a first interpretation). A different interpretation of the considered dynamics can also be given when $\xi(t)$ does not vanish on average. Then, for $\Delta_{+}>\Delta_{-}>0$, we are dealing with a quantum tunneling dynamics with a fluctuating tunneling matrix element, e.g., due to a fluctuating tunneling barrier.

For the considered case of a two state non-Markovian process with $p_{11}=p_{22}=0, p_{12}=p_{21}=1$ the general result in Eq. (18) can be simplified further. After some cumbersome operator algebra we obtain

$$
\begin{aligned}
\langle\tilde{S}(s)\rangle= & p_{+}^{\mathrm{st}} \tilde{S}_{+}(s)+p_{-}^{\mathrm{st}} \tilde{S}_{-}(s)-\frac{1}{\left\langle\tau_{+}\right\rangle+\left\langle\tau_{-}\right\rangle}\left\{\tilde{C}_{+}(s)+\tilde{C}_{-}(s)\right. \\
& -\left[\tilde{A}_{+}(s) \tilde{B}_{-}(s)+\tilde{A}_{-}(s)\right]\left[I-\tilde{B}_{+}(s) \tilde{B}_{-}(s)\right]^{-1} \tilde{A}_{+}(s) \\
& \left.-\left[\tilde{A}_{-}(s) \tilde{B}_{+}(s)+\tilde{A}_{+}(s)\right]\left[I-\tilde{B}_{-}(s) \tilde{B}_{+}(s)\right]^{-1} \tilde{A}_{-}(s)\right\},
\end{aligned}
$$

where $\tilde{S}_{ \pm}(s)$ denotes the Laplace-transform of the propagator $S_{ \pm}(t)=\exp \left(-\mathrm{i} \mathscr{L}_{ \pm} t\right)$ with $\mathscr{L}_{ \pm}:=\mathscr{L}\left[\Delta_{ \pm}\right]$corresponding to the fixed noise value $\xi=\Delta_{+}$and $\xi=\Delta_{-}$, correspondingly. Furthermore, $\tilde{C}_{ \pm}(s)$ is given by Eq. (21), $\tilde{C}_{ \pm}(s) \equiv \tilde{C}_{ \pm \pm}(s)$ and:

$\tilde{A}_{ \pm}(s):=\int_{0}^{\infty} \Phi_{ \pm}(\tau) \exp \left[-\left(s+\mathrm{i} \mathscr{L}_{ \pm}\right) \tau\right] \mathrm{d} \tau$,
$\tilde{B}_{ \pm}(s):=\int_{0}^{\infty} \psi_{ \pm}(\tau) \exp \left[-\left(s+\mathrm{i} \mathscr{L}_{ \pm}\right) \tau\right] \mathrm{d} \tau$.

A quantum evolution characterized in Eqs. (33)-(35) by the Liouville operators $\mathscr{L}_{ \pm}$does belong to a rather broad class (e.g., for quantum systems with a finite number of states) and is not merely restricted to the case of two-state quantum dynamics in Eq. (30).

The archetype model in Eq. (30) does exhibit a rich behavior. In particular, it opens a doorway to study the problem of quantum decoherence of a two-state atom under the influence of two-state " $1 / \omega^{\alpha}$ " noise that exhibits long range time-correlations with a power law decay (for $\psi(\tau)$ possessing a long-time algebraic tail, $\psi(\tau) \propto 1 / \tau^{3-\alpha}$, $0<\alpha<1)$ [72-74]. Therefore, this model constitutes a prominent problem of general interest. Moreover, it relates to recent activities that involve decoherence studies for solid state quantum computing [75]. It is convenient to express the Hamiltonian (30) in terms of Pauli matrices, $\hat{\sigma}_{z}:=|1\rangle\langle 1|-| 2\rangle\left\langle 2\left|, \quad \hat{\sigma}_{x}:=\right| 1\right\rangle\langle 2|+| 2\rangle\langle 1|, \quad \hat{\sigma}_{y}:=\mathrm{i}(|2\rangle\langle 1|-$ $|1\rangle\langle 2|)$ and the unit matrix $\hat{I}$,
$H(t)=\frac{1}{2} \hbar \epsilon_{0} \hat{\sigma}_{z}+\frac{1}{2} \hbar \xi(t) \hat{\sigma}_{x}+\frac{1}{2}\left(E_{1}+E_{2}\right) \hat{I}$,

where $\epsilon_{0}=\left(E_{1}-E_{2}\right) / \hbar$. The dynamics of the density matrix of the quantum two-state quantum system is then obtained as $\rho(t)=\frac{1}{2}\left[\hat{I}+\sum_{i=x, y, z} \sigma_{i}(t) \hat{\sigma}_{i}\right]$ with components $\sigma_{i}(t)=$ $\operatorname{Tr}\left(\rho(t) \hat{\sigma}_{i}\right)$. This latter dynamics evolves on a Bloch sphere of unit radius (i.e., the corresponding (scaled) magnetic moment is conserved, i.e., $\left.{ }^{3}|\vec{\sigma}(t)|=1\right)$. Its rate of change obeys:

$\dot{\sigma}_{x}(t)=-\epsilon_{0} \sigma_{y}(t)$,

$\dot{\sigma}_{y}(t)=\epsilon_{0} \sigma_{x}(t)-\xi(t) \sigma_{z}(t)$,

$\dot{\sigma}_{z}(t)=\xi(t) \sigma_{y}(t)$,

or $\dot{\vec{\sigma}}(t)=\hat{F}[\xi(t)] \vec{\sigma}(t)$ in the vector form, where

$\hat{F}[\xi(t)]=\left(\begin{array}{ccc}0 & -\epsilon_{0} & 0 \\ \epsilon_{0} & 0 & -\xi(t) \\ 0 & \xi(t) & 0\end{array}\right)$.

The above theory can readily be applied to the noise averaging of a 3-dimensional system of linear differential Eq. (37) over arbitrary stationary realizations of $\xi(t)$ with the obvious formal substitution $-\mathrm{i} \mathscr{L}[\xi(t)] \rightarrow F[\xi(t)]$. Towards this goal, we represent the propagators $\hat{S}_{ \pm}(t)=\exp \left(\hat{F}\left[\Delta_{ \pm}\right] t\right)$ for the fixed static values of noise $\xi=\Delta_{+}$and $\xi=\Delta_{-}$as matrix expansions over the eigenmodes of evolution $\exp \left(\mathrm{i} \lambda_{ \pm}^{(k)} t\right)$, with $\lambda_{ \pm}^{(0)}=0, \quad \lambda_{ \pm}^{(1)}=\Omega_{ \pm}, \quad \lambda_{ \pm}^{(2)}=-\Omega_{ \pm}$, where $\Omega_{ \pm}=\sqrt{\epsilon_{0}^{2}+\Delta_{ \pm}^{2}}$ are the eigenfrequencies of coherent quantum oscillations for constant $\xi=\Delta_{ \pm}$. It reads,

$\hat{S}_{ \pm}(t)=\sum_{k=0,1,2} \hat{R}_{ \pm}^{(k)} \exp \left(\mathrm{i} \lambda_{ \pm}^{(k)} t\right)$

where

$\hat{R}_{ \pm}^{(0)}=\frac{1}{\Omega_{ \pm}^{2}}\left(\begin{array}{ccc}\Delta_{ \pm}^{2} & 0 & \epsilon_{0} \Delta_{ \pm} \\ 0 & 0 & 0 \\ \epsilon_{0} \Delta_{ \pm} & 0 & \epsilon_{0}^{2}\end{array}\right)$,

$\hat{R}_{ \pm}^{(1)}=\left[\hat{R}_{ \pm}^{(2)}\right]^{*}=\frac{1}{2}\left(\begin{array}{ccc}\frac{\epsilon_{0}^{2}}{\Omega_{ \pm}^{2}} & \mathrm{i} \frac{\epsilon_{0}}{\Omega_{ \pm}} & -\frac{\epsilon_{0} \Delta_{ \pm}}{\Omega_{ \pm}^{2}} \\ -\mathrm{i} \frac{\epsilon_{0}}{\Omega_{ \pm}} & 1 & \mathrm{i} \frac{\Delta_{ \pm}}{\Omega_{ \pm}} \\ -\frac{\epsilon_{0} \Delta_{ \pm}}{\Omega_{ \pm}^{2}} & -\mathrm{i} \frac{\Delta_{ \pm}}{\Omega_{ \pm}} & \frac{\Delta_{ \pm}^{2}}{\Omega_{ \pm}^{2}}\end{array}\right)$,

$\sum_{k=0,1,2} \hat{R}_{ \pm}^{(k)}=\hat{I}$

The corresponding Laplace-transformed matrices entering Eq. (33) are represented as:

\footnotetext{
${ }^{3}$ This means that each and every stochastic trajectory runs on the Bloch sphere. The stochastically averaged Bloch vector dynamics $\langle\vec{\sigma}(t)\rangle$ is, however, "dissipative"; i.e., $|\langle\vec{\sigma}(t)\rangle| \leqslant 1$, because $\left\langle\sigma_{i}(t)\right\rangle^{2} \leqslant\left\langle\sigma_{i}^{2}(t)\right\rangle$. Thus, the averaged density matrix $\langle\rho(t)\rangle$ remains always positive in the considered model, independently of the particular model used for the stochastic force $\xi(t)$.
} 
$\tilde{S}_{ \pm}(s)=\sum_{k=0,1,2} \frac{\hat{R}_{ \pm}^{(k)}}{s-\mathrm{i} \lambda_{ \pm}^{(k)}}$

$\tilde{A}_{ \pm}(s)=\sum_{k=0,1,2} \hat{R}_{ \pm}^{(k)} \frac{1-\tilde{\psi}_{ \pm}\left(s-\mathrm{i} \lambda_{ \pm}^{(k)}\right)}{s-\mathrm{i} \lambda_{ \pm}^{(k)}}$

$\tilde{B}_{ \pm}(s)=\sum_{k=0,1,2} \hat{R}_{ \pm}^{(k)} \tilde{\psi}_{ \pm}\left(s-\mathrm{i} \lambda_{ \pm}^{(k)}\right)$,

$\tilde{C}_{ \pm}(s)=\sum_{k=0,1,2} \hat{R}_{ \pm}^{(k)} \frac{1-\tilde{\psi}_{ \pm}\left(s-\mathrm{i} \lambda_{ \pm}^{(k)}\right)}{\left(s-\mathrm{i} \lambda_{ \pm}^{(k)}\right)^{2}}$.

The results in Eqs. (33), (40), (41) form our basis for further studies.

Let us evaluate it explicitly for the case of symmetric process, $\psi_{+}(\tau)=\psi_{-}(\tau)=\psi(\tau),\left\langle\tau_{+}\right\rangle=\left\langle\tau_{-}\right\rangle=\langle\tau\rangle$, with zero mean value, $\Delta_{+}=-\Delta_{-}=\Delta$. We consider the situation where the state " 1 " is populated initially with the probability one: $\sigma_{z}(0)=1, \sigma_{x, y}(0)=0$. Then, the Laplace-transform of the averaged difference of two populations, i.e., $\left\langle\sigma_{z}(t)\right\rangle=\left\langle\rho_{11}(t)\right\rangle-\left\langle\rho_{22}(t)\right\rangle$ is denoted as $\left\langle\tilde{\sigma}_{z}(s)\right\rangle=\left\langle\tilde{S}_{z z}(s)\right\rangle$. After some cumbersome manipulations using a computer algebra system (MAPLE) to perform multiple matrix operations, we end up with the following compact result:

$\left\langle\tilde{\sigma}_{z}(s)\right\rangle=\frac{s^{2}+\epsilon_{0}^{2}}{s\left(s^{2}+\Omega^{2}\right)}-\frac{2 \Delta^{2}}{\langle\tau\rangle s^{2}\left(s^{2}+\Omega^{2}\right)^{2}} \frac{\tilde{A}_{z z}(s)}{\tilde{B}_{z z}(s)}$,

where

$$
\begin{aligned}
\tilde{A}_{z z}(s)= & \epsilon_{0}^{2}[1-\tilde{\psi}(s)]\left\{\left(\Omega^{2}-s^{2}\right)(1-\tilde{\psi}(s+\mathrm{i} \Omega) \tilde{\psi}(s-\mathrm{i} \Omega))\right. \\
& -2 \mathrm{i} \Omega s[\tilde{\psi}(s+\mathrm{i} \Omega)-\tilde{\psi}(s-\mathrm{i} \Omega)]\} \\
& -\Delta^{2} s^{2}[1+\tilde{\psi}(s)][1-\tilde{\psi}(s+\mathrm{i} \Omega)][1-\tilde{\psi}(s-\mathrm{i} \Omega)], \\
\tilde{B}_{z z}(s)= & \epsilon_{0}^{2}[1-\tilde{\psi}(s)][1+\tilde{\psi}(s+\mathrm{i} \Omega)][1+\tilde{\psi}(s-\mathrm{i} \Omega)] \\
& +\Delta^{2}[1+\tilde{\psi}(s)](1-\tilde{\psi}(s+\mathrm{i} \Omega) \tilde{\psi}(s-\mathrm{i} \Omega)) .
\end{aligned}
$$

Note that for the considered initial condition, we find $\left\langle\sigma_{x}(t)\right\rangle=\left\langle\sigma_{y}(t)\right\rangle=0$ for all times because $\left\langle\tilde{S}_{x z}(s)\right\rangle=$ $\left\langle\tilde{S}_{y z}(s)\right\rangle=0$. For $\epsilon_{0}=0$ the result in (42) and (43) reduces to one for Kubo oscillator (26) with identical $\psi_{1,2}(\tau)$. Moreover, for the Markovian case, $\tilde{\psi}(s)=1 /(1+\tau s)$, Eq. (42) reduces to

$\left\langle\tilde{\sigma}_{z}(s)\right\rangle=\frac{s^{2}+2 v s+v^{2}+\epsilon_{0}^{2}}{s^{3}+2 v s^{2}+\left(\Delta^{2}+\epsilon_{0}^{2}+v^{2}\right) s+\Delta^{2} v}$,

where $v=2 /\langle\tau\rangle$ is the inverse autocorrelation time. This latter result reproduces the result for the averaged populations $\left\langle\tilde{\rho}_{11}(s)\right\rangle=\left(1 / s+\left\langle\tilde{\sigma}_{z}(s)\right\rangle\right) / 2$ and $\left\langle\tilde{\rho}_{22}(s)\right\rangle=$ $\left(1 / s-\left\langle\tilde{\sigma}_{z}(s)\right\rangle\right) / 2$ in $[40,44]$. The same result (44) can also be deduced from the known solution for the Markovian case driven by asymmetric two-state noise [48] when specified to symmetric noise limit.

This population difference possesses several remarkable features: The asymptotic difference between populations is zero, $\left\langle\sigma_{z}(\infty)\right\rangle=\lim _{s \rightarrow 0}\left(s\left\langle\tilde{\sigma}_{z}(s)\right\rangle\right)=0$. In other words, the steady-state populations of both energy levels equal $1 / 2$, independent of the energy difference $\hbar \epsilon_{0}$. This result can be elucidated best in terms of a "temperature" $T_{\sigma}$ of the (pseudo-)spin system. This spin-temperature is formally introduced by using for the asymptotic distribution an Ansatz of the Boltzmann-Gibbs form, $\left\langle\rho_{n n}(\infty)\right\rangle=$ $\exp \left[-E_{n} / k_{\mathrm{B}} T_{\sigma}\right] / \sum_{n} \exp \left[-E_{n} / k_{\mathrm{B}} T_{\sigma}\right]$. Then, ${ }^{4}$

$T_{\sigma}:=\frac{\hbar \epsilon_{0}}{k_{\mathrm{B}} \ln \left(\frac{\left\langle\rho_{22}(\infty)\right\rangle}{\left\langle\rho_{11}(\infty)\right\rangle}\right)}$

for two-level systems. In accordance with this definition, the result of equal asymptotic populations, $\left\langle\rho_{22}(\infty)\right\rangle=\left\langle\rho_{11}(\infty)\right\rangle=1 / 2$ can be interpreted in terms of an infinite temperature $T_{\sigma}=\infty$. This constitutes a general finding: a purely stochastic bath corresponds to an apparently infinite temperature $[22,23]$. Thus, this stochastic approach modeling the relaxation process in open quantum systems is suitable only for sufficiently high temperatures $k_{\mathrm{B}} T \gg \hbar\left|\epsilon_{0}\right|[22,23]$. An asymmetry of unbiased stochastic fluctuations does not impact this conclusion, see in Ref. [48]. Moreover, the relaxation to the steady-state can be either coherent, or incoherent, depending on the noise strength and the value of autocorrelation time. In particular, an approximate analytical expression for the rate $k$ of incoherent relaxation, $\left\langle\rho_{11}(t)\right\rangle=[1+\exp (-k t)] / 2$, has been obtained in a limit of small Kubo numbers, $K:=\Delta / v \ll 1$, which corresponds to a weakly colored noise $[31,36]$. This analytic result reads $[40,44,48]$

$k=\frac{\Delta^{2} v}{v^{2}+\epsilon_{0}^{2}}$.

The rate exhibits a resonance feature vs. $v$ at $v=\epsilon_{0}$. A similar resonance feature occurs also in the theory of nuclear magnetic resonance for weakly colored Gaussian noise [76]. Note that in Ref. [48] this notable result has been obtained for asymmetric fluctuations of the tunneling coupling possessing a non-vanishing mean value $\langle\xi(t)\rangle \neq 0$. This corresponds to a quantum particle transfer between two sites of localization which are separated by a fluctuating tunneling barrier. A related problem with the inclusion of quantum dissipation has been elaborated in [52] within a stochastically driven spin-boson model.

Yet another interesting solution can be obtained for $\left\langle\tilde{\sigma}_{x}(s)\right\rangle$ with the initial condition reading $\sigma_{x}(0)=1$. The Laplace transform of the solution reads

$\left\langle\tilde{\sigma}_{x}(s)\right\rangle=\frac{s^{2}+\Delta^{2}}{s\left(s^{2}+\Omega^{2}\right)}-\frac{2 \Delta^{2} \epsilon_{0}^{2} \Omega^{2}}{\langle\tau\rangle s^{2}\left(s^{2}+\Omega^{2}\right)^{2}} \frac{\tilde{A}_{x x}(s)}{\tilde{B}_{x x}(s)}$,

where

$$
\begin{aligned}
\tilde{A}_{x x}(s)= & {[1-\tilde{\psi}(s)][1-\tilde{\psi}(s+\mathrm{i} \Omega)][1-\tilde{\psi}(s-\mathrm{i} \Omega)], } \\
\tilde{B}_{x x}(s)= & \epsilon_{0}^{2}[1+\tilde{\psi}(s)][1-\tilde{\psi}(s+\mathrm{i} \Omega)][1-\tilde{\psi}(s-\mathrm{i} \Omega)] \\
& +\Delta^{2}[1-\tilde{\psi}(s)](1-\tilde{\psi}(s+\mathrm{i} \Omega) \tilde{\psi}(s-\mathrm{i} \Omega)) .
\end{aligned}
$$

\footnotetext{
${ }^{4}$ This is the standard definition of the temperature of a spin subsystem in nuclear magnetic resonance and similar research areas [76]. It is used also to introduce the parlance of formally negative temperatures.
} 
The physical relevance of this solution (47) is as follows: In the rotated pseudo-spin basis, $\hat{\sigma}_{x} \rightarrow \hat{\sigma}_{z}, \hat{\sigma}_{z} \rightarrow \hat{\sigma}_{x}, \hat{\sigma}_{y} \rightarrow \hat{\sigma}_{y}$, this problem becomes mathematically equivalent to the problem of the delocalization of a quantum particle in a symmetric dimer with a tunneling coupling $\epsilon_{0}$ under the influence of a dichotomously fluctuating energy bias $\xi(t)$. Therefore, it describes the corresponding delocalization dynamics and, in particular, allows one to determine whether this dynamics is coherent or incoherent, depending on the noise characteristics.

For the Markovian case, Eq. (47) reduces to ${ }^{5}$

$\left\langle\tilde{\sigma}_{x}(s)\right\rangle=\frac{s^{2}+v s+\Delta^{2}}{s^{3}+v s^{2}+\left(\Delta^{2}+\epsilon_{0}^{2}\right) s+\epsilon_{0}^{2} v}$.

Note that the denominators in Eqs. (44) and (49) are different. ${ }^{6}$ In the more general case of asymmetric Markovian noise, the corresponding denominator is a polynomial of sixth order in $s$, see [48]. In the considered case of symmetric noise it factorizes into the product of two polynomials of third order, namely into those in the denominators of Eqs. (44) and (49). Thus, for a general initial condition the relaxation of a two-level quantum system exposed to a two-state Markovian field involves six exponential terms. As a matter of fact, this seemingly simple, exactly solvable model can exhibit an unexpectedly complex behavior even in the Markovian case of a colored two-state noise. However, for certain initial conditions, as exemplified above, the general solution being a fraction of two polynomials of $s$ simplifies to the results in Eqs. (44) and (49).

In a general case of non-Markovian noise, the analytical solutions in Eqs. (42) and (47) can be inverted numerically to the time domain by use of a numerical Laplace inversion procedure such as the one detailed in Ref. [77] using a computer algebra implementation with arbitrary digital precision [78]. A quasi-analytical inversion is, however, still possible in specific non-Markovian cases.

\section{A simple non-Markovian case}

We consider the following basic case of non-Markovian noise described by a biexponential RTD

$\psi(\tau)=\theta \alpha_{1} \exp \left(-\alpha_{1} \tau\right)+(1-\theta) \alpha_{2} \exp \left(-\alpha_{2} \tau\right)$,

\footnotetext{
${ }^{5}$ The corresponding dynamics also exhibits a resonance feature vs. the inverse autocorrelation time $v$ within certain limits [50].

${ }^{6}$ A notable feature is, however, that both corresponding secular cubic equations have the same discriminant, $D\left(\Delta, v, \epsilon_{0}\right)=0$, separating the domains of complex and real roots. Hence, the transition from a coherent relaxation (i.e., complex roots are present) to an incoherent relaxation (i.e., only real roots are obtained) occurs at the same values of noise parameters, independently of the initial conditions. The corresponding phase diagram separating regimes of coherent and incoherent relaxation (judging from the above criterion) has been devised in [50]. It must be kept in mind, however, that the weights of the corresponding exponentials are also of importance for the characteristic relaxation dynamics. These weights do depend on the initial conditions.
}

where $\alpha_{1,2}$ denote two transition rates which can be realized with probabilities $\theta$ and $1-\theta$, correspondingly. The considered noise possesses the mean residence time

$\langle\tau\rangle=\theta / \alpha_{1}+(1-\theta) / \alpha_{2}$

and the mean autocorrelation time $\tau_{\text {corr }}:=\int_{0}^{\infty}|k(t)| \mathrm{d} t$ reading with $k(t) \geqslant 0$,

$\tau_{\text {corr }}=C_{V}^{2} \tau_{\text {corr }}^{(M)}$,

where $C_{V}=\sqrt{\left\langle\tau^{2}\right\rangle-\langle\tau\rangle^{2}} /\langle\tau\rangle$ is the coefficient of variation of the RTD $[73,74]$ and $\tau_{\text {corr }}^{(M)}=\langle\tau\rangle / 2$ is the autocorrelation time of Markovian process possessing the same mean residence time $\langle\tau\rangle$. The ratio $\tau_{\text {corr }} / \tau_{\text {corr }}^{(M)}=C_{V}^{2}$ serves as a convenient quantifier for non-Markovian effects. For this basic non-Markovian case considered, we find that $C_{V}^{2}$ can be large, see in Ref. [64] for details. For example, in the limit $\zeta=\alpha_{1} / \alpha_{2} \ll \theta \ll 1, C_{V}^{2} \approx 2 / \theta \gg 1$. Such a noise has distinct bursting features [64].

For this noise, Eq. (42) reduces to a rational function of $s$,

$\left\langle\tilde{\sigma}_{z}(s)\right\rangle=\frac{N_{z z}(s)}{D_{z z}(s)}$,

where $N_{z z}(s)$ and $D_{z z}(s)$ are specific polynomials of fifth and sixth orders, correspondingly. The explicit form of these polynomials is not given here since it does not provide much physical insight.

The inversion of (53) to the time domain reads

$\left\langle\sigma_{z}(t)\right\rangle=\sum_{k=1}^{6} \frac{N_{z z}\left(r_{k}\right)}{D_{z z}^{\prime}\left(r_{k}\right)} \exp \left(r_{k} t\right)$

where $r_{k}$ are the complex roots $\left(\operatorname{Re} r_{k}<0\right)$ of $D_{z z}(s)=0$ and $D_{z z}^{\prime}(s):=\mathrm{d} D_{z z}(s) / \mathrm{d} s$. Even if $r_{k}$ cannot be determined analytically (except for the case $\epsilon_{0}=0$, where the considered rational function can be simplified to the ratio of two polynomials of the third and fourth orders, correspondingly), they can be found numerically for any set of parameters entering the problem. The problem is thereby quasi-analytically solvable. The same is valid for taking more terms in the exponential expansion of $\psi(\tau)$. The whole scheme can be easily implemented using a computer algebra system like MAPLE or MATHEMATICA. In this respect, it is pertinent to note that a power law dependence can be well approximated by an expansion of the type $\psi(\tau)=$ $\sum_{i} c_{i} \alpha_{i} \exp \left(-\alpha_{i} \tau\right)$. Indeed, a power law extending over $n$ decades can be satisfactory approximated already by an $n$-term expansion with properly scaled $\left\{\alpha_{i}\right\}$ and $\left\{c_{i}\right\}$ [79]; see also a practical example of a 6-exponential fitting in [80]. Moreover, the numerical procedures of the Laplace-transform inversion like one in [78] can be implemented. An analytical inversion of Eq. (47) to the time-domain has the form similar to Eq. (54), but with different polynomials $N_{x x}(s)$ and $D_{x x}(s)$ of fifth and sixth order, correspondingly.

\subsection{Numerical results}

We consider first the situation with a small Kubo number $K=\Delta \tau_{\text {corr }} \ll 1$ where an approximate analytical result, 
$\left\langle\sigma_{z}(t)\right\rangle=\exp (-k t)$, is available with the rate $k$ in Eq. (46) $[44,48]$. This result can also be obtained using the cumulant expansion method [31]. It does not depend on whether is the stochastic process under consideration is Markovian or not. This is illustrated with Fig. 1. The following parameters are used in calculations presented in Fig. 1 (units are arbitrary): energy difference between levels $\epsilon_{0}=1$, noise amplitude $\Delta=0.5$. Furthermore, for the Markovian twostate noise we have chosen: $\alpha_{1}=100$ (here $\theta=1$ ) and for the non-Markovian one: $\alpha_{1}=100, \quad \alpha_{2}=2000$, $\theta \approx 0.05263157894$. Both noises possess the same autocorrelation time $\tau_{\text {corr }}=0.005$ while the average residence times differ by an order of magnitude; the non-Markovian parameter is $C_{V}^{2}=10$. On the characteristic time scale of quantum relaxation the relaxation process in both cases is excellently described by the approximate analytical result given above. All three lines practically coincide in Fig. 1. In this case, the perturbation theory in the small Kubo number $K$ works very well.

It is interesting to note that the initial decay of populations is (beyond the discussed exponential approximation) always Gaussian, i.e., $\ln \left\langle\sigma_{z}(t)\right\rangle \propto-t^{2}$ at $t \rightarrow 0$. This "Gaussian" regime can be, however, very short and, therefore, it is not readily visible on the characteristic time scale of the relaxation like in Fig. 1.

In the opposite limit, $K \gg 1$, the distinction between the influence of Markovian and non-Markovian noises possessing the same (mean) autocorrelation time becomes rather distinct, cf. Fig. 2. Here, the following noise parameters are used: $\Delta=0.5, \alpha_{1}=0.05, \theta=1$ (for the Markovian noise); $\alpha_{1}=0.05, \alpha_{2}=1, \theta \approx 0.05263157894$ (for the nonMarkovian noise). In both cases, the autocorrelation time is the same $\tau_{\text {corr }}=10$, while the mean residence times are

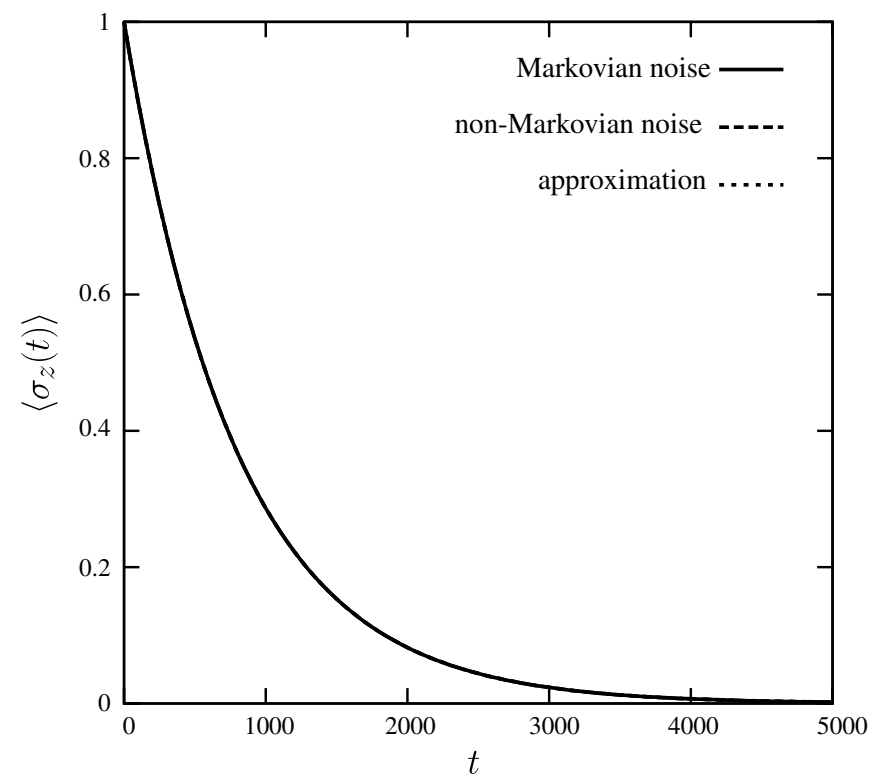

Fig. 1. Quantum relaxation in the perturbative regime. All three curves practically coincide on the considered time scale, see text for details.

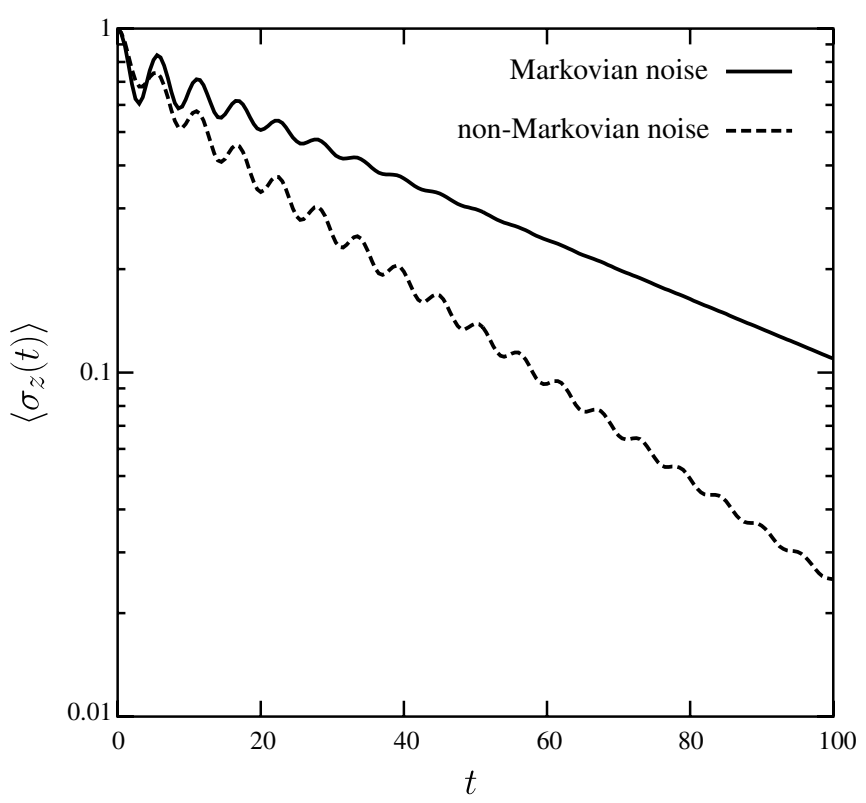

Fig. 2. Quantum relaxation in the non-perturbative regime of a large Kubo number, $K=10$. The influence of Markovian and non-Markovian noises with the same rms and mean autocorrelation time are distinctly different. Coherent features are more pronounced for the non-Markovian noise case. Note that in the latter case the overall relaxation time is shorter.

quite different: $\langle\tau\rangle=20$ for the Markovian case and $\langle\tau\rangle=2$ for the non-Markovian case.

\section{The manifest non-Markovian case}

For a situation in which the autocorrelation time of noise $\tau_{\text {corr }}$ diverges, the corresponding Kubo number $K=\Delta \tau_{\text {corr }}$ is infinite and any perturbation theory is doomed to fail. The theory developed herein, however, allows to obtain convergent numerical results by performing the inverse Laplace transformation numerically. We illustrate the strength of our theory with the following example of manifest non-Markovian noise with an RTD given by

$\tilde{\psi}(s)=\frac{1}{1+s\langle\tau\rangle g_{\alpha}\left(s \tau_{\mathrm{d}}\right)}$,

where

$g_{\alpha}(z)=\frac{\tanh \left(z^{\alpha / 2}\right)}{z^{\alpha / 2}}$

with $0<\alpha<1$. Here, $\langle\tau\rangle$ in Eq. (55) is the mean residence time and $\tau_{\mathrm{d}}$ is a time constant presenting an additional parameter of the distribution. For $\tau_{\mathrm{d}}=0$, an exponential distribution is restored. The properties of the RTD determined by Eqs. (55) and (56) are discussed in detail in [81]. This RTD thus possesses three parameters only, but it exhibits an interesting repertoire of effects. In particular, it can encompass up to three different interchanging power law regimes with the asymptotic power law assuming the form $\psi(\tau) \propto 1 / \tau^{2+\alpha}, \tau \rightarrow \infty[81]$. 
The corresponding noise has $1 / \omega^{1-\alpha}$ feature in its power spectrum at $\omega \rightarrow 0$. Its mean autocorrelation time $\tau_{\text {corr }}$ diverges, $\tau_{\text {corr }}=\infty$. Therefore, the Kubo number $K$ is formally infinite and a corresponding perturbation theory is questionable. Our theory delivers, however, exact result for the Laplace-transformed noise-averaged quantum relaxation of the excited level population. Moreover, the mean relaxation time can be formally defined as $\tau_{\text {rel }}:=\int_{0}^{\infty}\left\langle\sigma_{z}(t)\right\rangle \mathrm{d} t$. With Eq. (42), this time follows as $\tau_{\text {rel }}=\lim _{s \rightarrow 0}\left\langle\tilde{\sigma}_{z}(s)\right\rangle$ for any function $\tilde{\psi}(s)$. Moreover, the asymptotic character of relaxation dynamics as $t \rightarrow \infty$ can be found by using the Tauberian theorems of the Laplace-transform method and small-s expansion of $\tilde{\psi}(s)$ reading (in leading terms)

$\tilde{\psi}(s) \approx 1-\langle\tau\rangle s+A s^{1+\alpha}$

for any $\psi(\tau)$ possessing the finite mean value $\langle\tau\rangle$ and the long-time algebraic tail $\psi(\tau) \propto 1 / \tau^{2+\alpha}$. For the particular case in Eq. (55), $A=\langle\tau\rangle \tau_{\mathrm{d}}^{\alpha} / 3$.

Using (57) in Eq. (42), after some algebra we obtained in the leading order:

$\left\langle\tilde{\sigma}_{z}(s)\right\rangle \sim \frac{A \epsilon_{0}^{2}}{\Omega^{2}\langle\tau\rangle} s^{\alpha-1}, \quad s \rightarrow 0$.

Several remarkable results follow readily. First, $\left\langle\sigma_{z}(\infty)\right\rangle=\lim _{s \rightarrow 0}\left[s\left\langle\tilde{\sigma}_{z}(s)\right]=0\right.$, i.e., the general drawback of the stochastic Liouville approach is preserved. Second, the use of a Tauberian theorem [56] in the above equation yields

$\left\langle\sigma_{z}(t)\right\rangle \sim \frac{A \epsilon_{0}^{2}}{\Omega^{2} \Gamma(1-\alpha)\langle\tau\rangle} \frac{1}{t^{\alpha}}, \quad t \rightarrow \infty$.

For the case in (55), this latter equation is modified as

$\left\langle\sigma_{z}(t)\right\rangle \sim \frac{\epsilon_{0}^{2}}{3 \Omega^{2} \Gamma(1-\alpha)}\left(\frac{\tau_{\mathrm{d}}}{t}\right)^{\alpha}, \quad t \rightarrow \infty$.

Remarkably, this result does not depend on the mean residence time $\langle\tau\rangle$. The tail of the relaxation curve clearly exhibits a power law, $\left\langle\sigma_{z}(t)\right\rangle \propto 1 / t^{\alpha}$.

The exact result for the Laplace-transformed relaxation in Eq. (42) can be reliably inverted numerically due a generalization of the well-known Stehfest method [77] in Ref. [78] which requires implementing this method, for example, with a computer algebra system like MAPLE (done here) using a sufficiently high digital precision. As a "rule of thumb" the number of digits $N$ used in our calculations should correspond to the number of terms taken in the Stehfest asymptotical series expansion [77]. $N$ must be increased until the numerical results converge with the required accuracy. For example, to obtain numerical data for Fig. 3 we used $N=256$. In this figure, the results are numerically precise within the corresponding line width. Note that the standard choice $N=16$ [77] is inadequate to obtain the correct numerical results for the averaged relaxation in Fig. 3. Following the reasoning in [78] we checked and confirmed these numerical considerations for
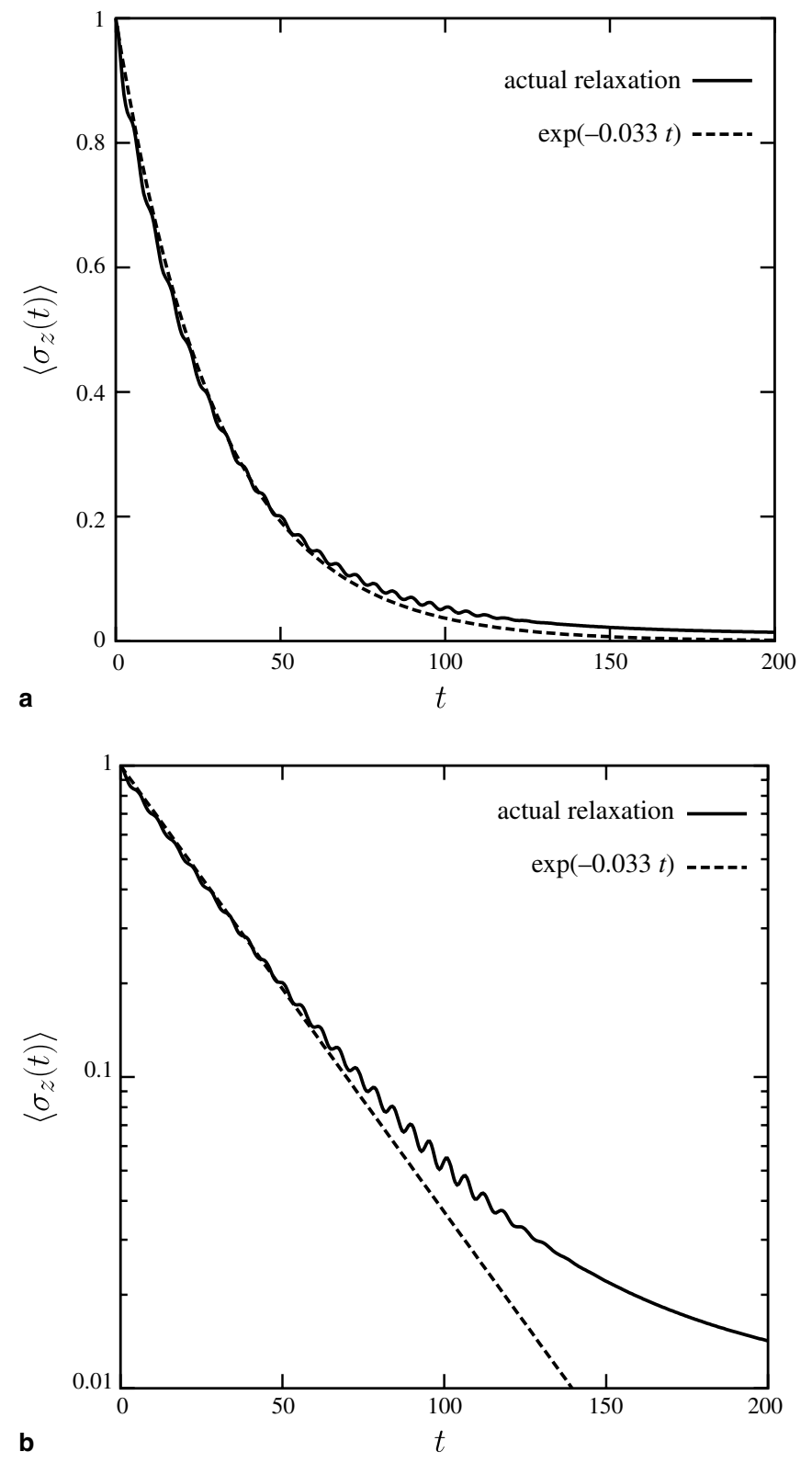

Fig. 3. (a) Quantum relaxation under the influence of non-Markovian two state noise with extreme long-time correlations. The parameters of this manifest non-Markovian two-state noise are: $\Delta=0.5, \alpha=0.5,\langle\tau\rangle=0.01$, $\tau_{\mathrm{d}}=1$. (b) The same data presented on log-linear scales.

some test functions with known results for the "function" - and its known Laplace-transform. Such an improved Stehfest method presents one of the best numerical Laplace transform inversion methods available nowadays (the core of the MAPLE code contains just a few lines, by the way).

For the results in Fig. 3 the following parameters have been used: $\epsilon_{0}$ and $\Delta$ are the same as in Figs. 1 and 2, i.e., $\epsilon_{0}=1$ and $\Delta=0.5 ; \tau=0.01$ (like for the Markovian case in Fig. 1), $\alpha=0.5$ (" $1 / \omega^{0.5}$ " noise) and $\tau_{\mathrm{d}}=1$. It is interesting to compare this case with those in Figs. 1 and 2. Surprisingly, the relaxation dynamics turns out to be initially practically a single exponential with small-amplitude quantum coherent oscillations superimposed. About $90 \%$ of the 


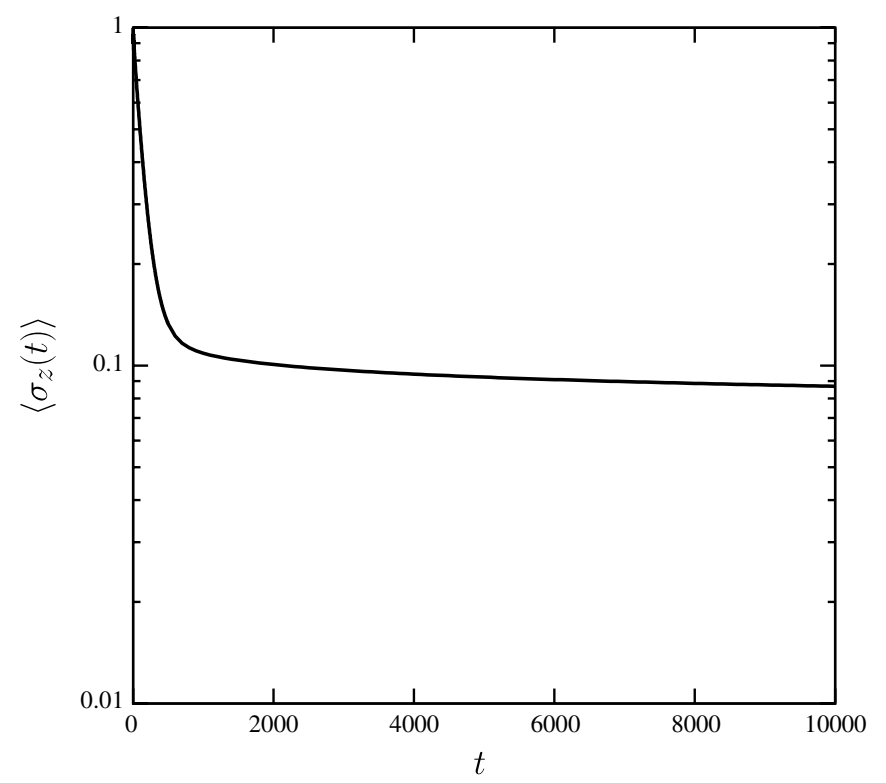

Fig. 4. Quantum relaxation under the influence of non-Markovian twostate noise with a $1 / \omega^{0.9}$ feature for its power spectrum. $\alpha=0.1$, the remaining parameters are the same as in Fig. 3.

initial population difference decays exponentially. The long time tail of the relaxation process is, however, clearly nonexponential, and the approach to the steady-state occurs much slowly than the initial exponential decay. The emergence of such a slow non-exponential asymptotic decay is rather intriguing. It is due to a manifest non-Markovian character of the noise as detailed analytically above. The same reasoning holds true is valid for $\alpha=0.1$; the corresponding noise obeys an $1 / \omega^{0.9}$ feature in its power spectrum which is close to $1 / f$ noise. In this case, the tail of relaxation curve becomes, however, more flat, being in accordance with Eq. (60), and the approach to the steady-state $\left\langle\sigma_{z}(\infty)\right\rangle=0$ occurs extremely slow: This might create an incorrect impression that $\left\langle\sigma_{z}(\infty)\right\rangle \neq 0$, see Fig. 4. We remark that for this particular case the digital precision $N=32$ was sufficient to obtain convergent results within the improved Stehfest method.

\section{Resume}

With this work we have presented general results for the averaged quantum relaxation that is driven by discrete state non-Markovian noise of the renewal, or CTRW type. The noise sources present non-Markovian generalization of discrete state Markovian noise sources. Our focus has been on the averaged time-evolution in presence of stationary noise realizations (i.e., the noise does not relax to, but is in its steady-state while the noise-averaged quantum dynamics undergoes a relaxation process). The practical feasibility of our approach has been elucidated with several applications of general interest, namely (i) the averaging of the Kubo oscillator (for arbitrary noises) and (ii) the averaged relaxation dynamics of a quantum two state sys- tem that is driven by two state non-Markovian noise. For this latter case and for a symmetric process, tractable analytical expressions have been obtained in Eqs. (42) and (47). The previously known results for the case of Markovian noise are recovered from our more general expressions. These new analytical results have been verified by corresponding numerical studies. In particular, the case of a manifest non-Markovian noise possessing an infinite variance of the residence time distribution, an infinite mean autocorrelation time and an $1 / \omega^{\alpha}$ feature in its power spectrum has been studied. This latter situation cannot be tackled within perturbation theory. However, our theory allows for a definite non-perturbative treatment. The authors share the confident belief that this new theory will prove useful for this and similar future investigations of quantum relaxation processes that are exposed to one or several noisy environments exhibiting characteristic longtime memory.

\section{Acknowledgment}

This work has been supported in parts by the Deutsche Forschungsgemeinschaft within the collaborative research centre, SFB-486: "Manipulation of matter on the nanoscale", project A10.

\section{References}

[1] R.A. Marcus, J. Chem. Phys. 24 (1956) 966.

[2] N.S. Hush, J. Chem. Phys. 28 (1958) 962.

[3] V.G. Levich, R.R. Dogonadze, Dokl. Acad. Nauk SSSR, Ser. Fiz. Khim. 124 (1959) 123 (in Russian).

[4] P. Hänggi, P. Talkner, M. Borkovec, Rev. Mod. Phys. 62 (1990) 251.

[5] M. Grifoni, P. Hänggi, Phys. Rep. 304 (1998) 229.

[6] S. Kohler, J. Lehmann, P. Hänggi, Phys. Rep. 406 (2005) 379.

[7] J. Jortner, M. Bixon (Eds.), Electron Transfer - From Isolated Molecules to Biomolecules, Adv. Chem. Phys., 107, Wiley, New York, 1999.

[8] V. May, O. Kühn, Charge and Energy Transfer Dynamics in Molecular Systems, second ed., Wiley-VCH, Berlin, 2004.

[9] E.G. Petrov, Physics of Charge Transfer in Biosystems, Naukova Dumka, Kiev, 1984 (in Russian).

[10] I. Goychuk, P. Hänggi, Adv. Phys. 54 (2005).

[11] P.W. Anderson, P.R. Weiss, Rev. Mod. Phys. 25 (1953) 269.

[12] R. Kubo, J. Phys. Soc. Jpn. 9 (1954) 935.

[13] R. Kubo, in: D. ter Haar (Ed.), Fluctuation, Relaxation, and Resonance in Magnetic Systems, Oliver and Boyd, Edinburgh, 1962.

[14] R. Kubo, J. Math. Phys. 4 (1963) 174.

[15] R. Kubo, Adv. Chem. Phys. 15 (1969) 101.

[16] A.I. Burshtein, Zh. Eksp. Teor. Phys. 49 (1965) 1362 [Sov. Phys. JETP 22 (1966) 939].

[17] H. Haken, P. Reineker, Z. Phys. 249 (1972) 253.

[18] H. Haken, G. Strobl, Z. Phys. 262 (1973) 135.

[19] A. Blumen, R. Silbey, J. Chem. Phys. 69 (1978) 3589.

[20] R.F. Fox, Phys. Rep. 48 (1978) 181.

[21] S. Mukamel, I. Oppenheim, J. Ross, Phys. Rev. A 17 (1978) 1988.

[22] P. Reineker, V. Kenkre, P. Reineker, Exciton Dynamics in Molecular Crystals and Aggregates, Springers Tracts in Modern Physics, vol. 94, Springer, Berlin, 1982.

[23] K. Lindenberg, B. West, The Nonequilibrium Statistical Mechanics of Open and Closed Systems, VCH, New York, 1990.

[24] J.T. Stockburger, H. Grabert, Chem. Phys. 268 (2001) 249.

[25] J. Shao, J. Chem. Phys. 120 (2004) 5053. 
[26] A.A. Ovchinnikov, N.S. Erikhman, Zh. Exsp. Teor. Phys. 67 (1974) 1474 (in Russian) [Sov. Phys. JETP 40 (1975) 733].

[27] Y. Kayanuma, J. Phys. Soc. Jpn. 53 (1984) 108.

[28] Y. Kayanuma, J. Phys. Soc. Jpn. 54 (1985) 2037.

[29] J. Shao, C. Zerbe, P. Hänggi, Chem. Phys. 235 (1998) 81.

[30] P. Hänggi, P. Jung, Adv. Chem. Phys. 89 (1995) 239.

[31] N.G. Van Kampen, Stochastic Processes in Physics and Chemistry, second ed., North-Holland, Amsterdam, 1992.

[32] R. Bittl, K. Schulten, J. Chem. Phys. 90 (1989) 1794.

[33] U. Frisch, A. Brissaud, J. Quant. Spectrosc. Radiat. Transf. 11 (1971) 1753.

[34] N. Wiener, Ann. Math. 22 (1920) 66.

[35] R.P. Feynman, A.R. Hibbs, Quantum Mechanics and Path Integrals, McGraw-Hill, New York, 1965.

[36] A. Brissaud, U. Frisch, J. Math. Phys. 15 (1974) 524.

[37] P. Hänggi, Z. Physik B 31 (1978) 407.

[38] P. Hanggi, Z. Physik B 36 (1980) 271.

[39] P. Hanggi, Z. Physik B 43 (1981) 269.

[40] V.E. Shapiro, V.M. Loginov, Dynamical Systems under Stochastic Perturbations: Simple Methods of Analysis, Nauka, Novosibirsk, 1983 (in Russian).

[41] V.I. Klyatskin, Radiophys. Quant. Electron. 20 (1978) 382 [Izv. Vyssh. Ucheb. Zaved., Radiophyz. 20 (1977) 562].

[42] V.E. Shapiro, V.M. Loginov, Physica A 91 (1978) 563.

[43] P. Hänggi, in: L. Pesquera, M.A. Rodriguez (Eds.), Stochastic Processes Applied to Physics, World Scientific, Singapore, 1985, p. 69.

[44] M. Auvergne, A. Pouquet, Physica 66 (1973) 409.

[45] V. Kraus, P. Reineker, Phys. Rev. A 43 (1991) 4182.

[46] P. Reineker, Ch. Warns, Th. Neidlinger, I. Barvik, Chem. Phys. 177 (1993) 713.

[47] I.A. Goychuk, E.G. Petrov, V. May, Phys. Rev. E 51 (1995) 2982.

[48] E.G. Petrov, I.A. Goychuk, V. Teslenko, V. May, Phys. Lett. A 218 (1996) 343.

[49] J. Iwaniszewski, Phys. Rev. E 61 (2000) 4890.

[50] J. Ankerhold, P. Pechukas, Europhys. Lett. 52 (2000) 264.

[51] I. Barvik, C. Warns, T. Neidlinger, P. Reineker, Chem. Phys. 240 (1999) 173.

[52] I.A. Goychuk, E.G. Petrov, V. May, J. Chem. Phys. 103 (1995) 4937.

[53] N.G. van Kampen, Physica A 96 (1979) 435.

[54] A.I. Burshtein, A.A. Zharikov, S.I. Temkin, Theor. Math. Phys. 66 (1986) 166.
[55] P. Chvosta, P. Reineker, Physica A 268 (1999) 103.

[56] W. Feller, second corrected ed.An Introduction to Probability Theory and its Applications, vol. II, Wiley, New York, 1966.

[57] I. Goychuk, Phys. Rev. E 70 (2004) 016109.

[58] E.W. Montroll, G.H. Weiss, J. Math. Phys. 6 (1965) 167.

[59] M. Lax, H. Scher, Phys. Rev. Lett. 39 (1977) 781.

[60] M.F. Shlesinger, Encyclopedia of Applied Physics, vol. 16, VCH, New York, 1996, pp. 45-70.

[61] B.D. HughesRandom Walks and Random Environments, vol. 1, Clarendon Press, Oxford, 1995.

[62] D.R. Cox, Renewal Theory, Methuen, London, 1962.

[63] Y. Jung, E. Barkai, R.J. Silbey, Adv. Chem. Phys. 123 (2002) 199.

[64] I. Goychuk, J. Chem. Phys. 122 (2005) 164506.

[65] Y. Jung, E. Barkai, R.J. Silbey, Chem. Phys. 284 (2002) 181.

[66] E.G. Petrov, V.I. Teslenko, I.A. Goychuk, Phys. Rev. E 49 (1994) 3894.

[67] I. Goychuk, M. Grifoni, P. Hänggi, Phys. Rev. Lett. 81 (1998) 649;

I. Goychuk, M. Grifoni, P. Hänggi, Phys. Rev. Lett. 81 (1998) 2837 (erratum).

[68] P.D. Reilly, J.L. Skinner, J. Chem. Phys. 101 (1994) 959.

[69] E. Geva, J.L. Skinner, Chem. Phys. Lett. 288 (1998) 225.

[70] E. Barkai, R. Silbey, G. Zumofen, Phys. Rev. Lett. 84 (2000) 5339.

[71] R.L. Stratonovich, Topics in the Theory of Random Noise, vol. I, Gordon \& Breach, New York, 1963, p. 176.

[72] S.B. Lowen, M.C. Teich, Phys. Rev. E 47 (1993) 992.

[73] I. Goychuk, P. Hänggi, Phys. Rev. Lett. 91 (2003) 070601.

[74] I. Goychuk, P. Hänggi, Phys. Rev. E 69 (2004) 021104.

[75] H. Gutmann, F.K. Wilhelm, W.M. Kaminsky, S. Loyd, Phys. Rev. A 71 (2005) 020302.

[76] C.P. Slichter, Principles of Magnetic Resonance, Springer, Berlin, 1978.

[77] H. Stehfest, Comm. ACM 13 (1970) 47; H. Stehfest, Comm. ACM 13 (1970) 624 (Erratum).

[78] P.P. Valko, S. Vajda, Inverse Prob. Eng. 10 (2002) 467.

[79] R. Metzler, J. Klafter, J. Jortner, Proc. Natl. Acad. Sci. USA 96 (1999) 11085.

[80] M.S.P. Sansom, F.G. Ball, C.J. Kerry, R. McGee, R.L. Ramsey, P.N.R. Usherwood, Biophys. J. 56 (1989) 1229.

[81] I. Goychuk, P. Hänggi, Phys. Rev. 70 (2004) 051915. 